\title{
Management of class II div.2 malocclusion using TADs supported frog appliance
}

\begin{abstract}
This case report describes the effect of Temporary Anchorage Devices Supported Frog Appliance on Class II div.2. The use of miniscrews supported distalizer minimized unwanted teeth movement while distalizing maxillary molars and reduce the need of patient compliance. The design of appliance and treatment steps were demonstrated in this case report. Case selection is an important factor of molar distaliztion and achieving planed result.
\end{abstract}

Keywords: miniscrews, distalizer, class II div.2, anchorage, molar distalization
Volume 10 Issue 6 - 2019

\author{
Abdullah Mohammad Aldayel,' Ahmed Ali \\ Alfawzan' \\ 'Dental Department, King Saud University Medical City, Saudi \\ Arabia \\ ${ }^{2}$ Department of Preventive Dentistry,Alrass Dental College, \\ Qassim University, Saudi Arabia
}

\begin{abstract}
Correspondence: Ahmed Ali Alfawzan, Department of Preventive Dentistry, Alrass Dental College, Qassim University, Saudi Arabia, Qassim, Saudi Arabia, +966553936688,

Email dr.ahmed.alfawza@qudent.org
\end{abstract}

Received: November 22, 2019 | Published: December 02 2019 

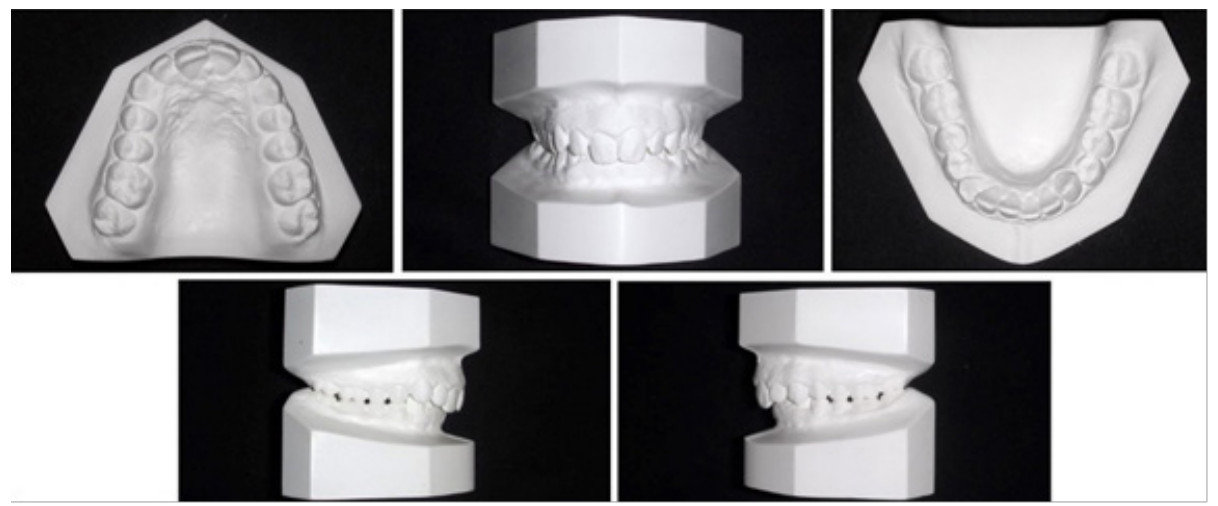

Figure 2 Pretreatment study models.
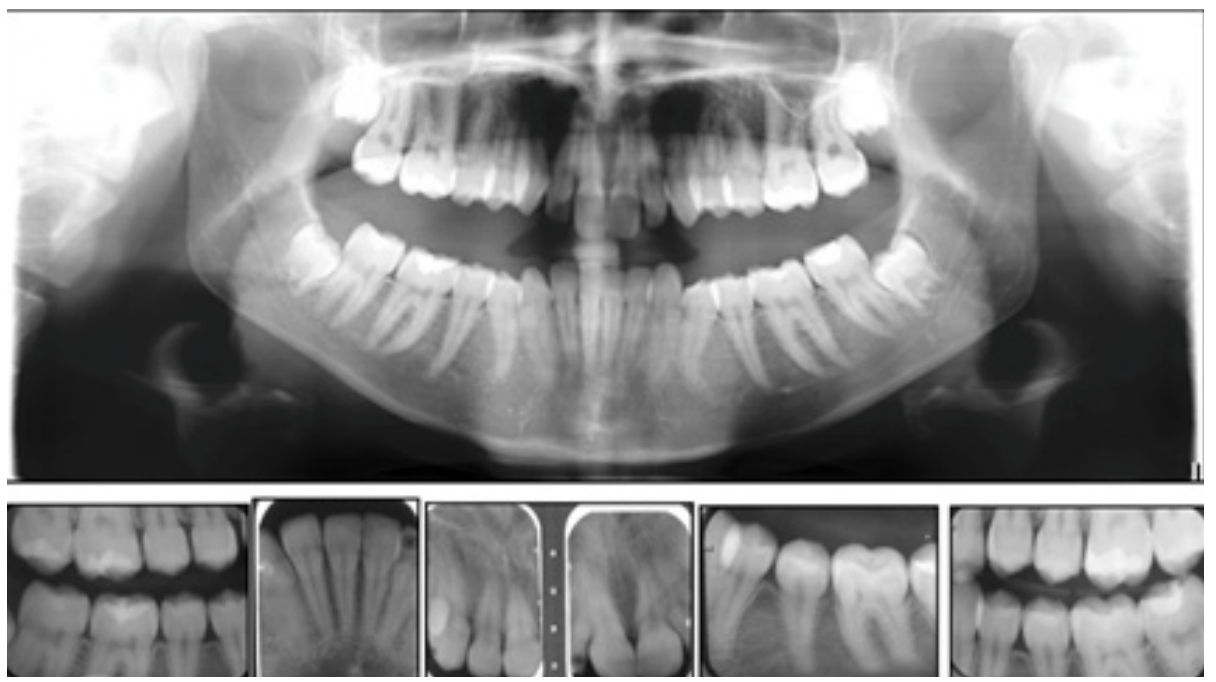

Figure 3 Pretreatment panoramic, periapical and bitewing radiographs.

Cephalometric analysis showed that patient had SNA angle of $80^{\circ}$, SNB angle of $76^{\circ}$, ANB angle of $4^{\circ}$ and MP angle of $26^{\circ}$. The upper incisors had a $102^{\circ}$ angle relative to the palatal plane and the lower incisors had a $90^{\circ}$ angle relative to the mandibular plane (Figure 4 \& Table 1).
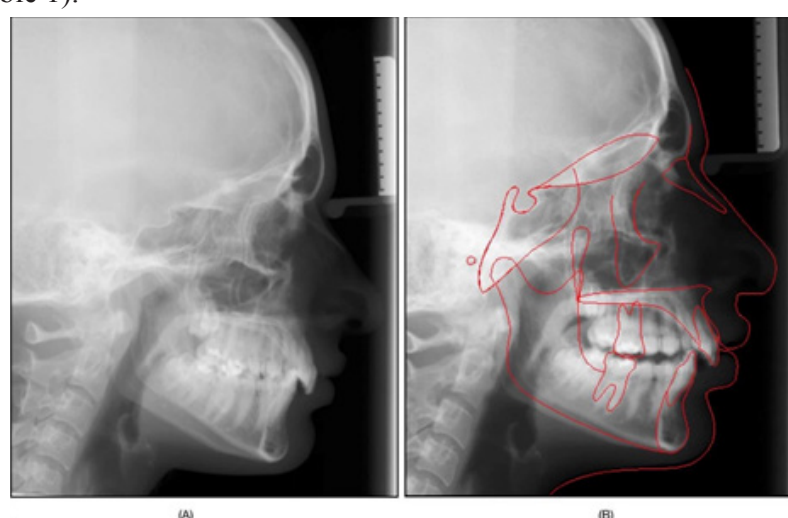

Figure 4 (A) Pretreatment Lateral Cephalometric Radiograph. Pretreatment Traced Lateral Cephalometric Radiograph.

Table I Pre-treatment lateral cephalometric reading

\begin{tabular}{lll} 
Measurement & Mean & Pre-treatment \\
\hline SNA & $82 \pm 2^{\circ}$ & $80.9^{\circ}$ \\
SNB & $80 \pm 2^{\circ}$ & $76.1^{\circ}$
\end{tabular}

ANB

$\mathrm{NA}-\mathrm{APg}$.

Wits Appraisal

Mand. plane to $\mathrm{FH}$

Mand. Plane to SN

Max. Plane to SN

Max. to Mand. Plane

$Y$ axis SGn. / SN

Lower Face Height

U Inc. to Max. plane

U Inc. to SN

$U$ Inc. to NA

$U$ Inc. to $N A(\mathrm{~mm})$

U Inc. to L Inc.

L Inc. to Mand.

$L$ Inc. to NB

L Inc. to NB (mm)

$L$ Inc. to APog. (mm)

Upper lip to E-line

Lower lip to E-line

Nasio-labial angle

$\begin{array}{ll}2 \pm 2^{\circ} & 4.8^{\circ} \\ 0 \pm 5^{\circ} & 6.1^{\circ} \\ 1.2 \pm 1.9 \mathrm{~mm} & 3.5 \mathrm{~mm} \\ 25 \pm 5^{\circ} & 19.3 \\ 32 \pm 5.1^{\circ} & 26.4 \\ 8 \pm 3^{\circ} & 10.3^{\circ} \\ 25 \pm 3^{\circ} & 21.6^{\circ} \\ 59 \pm 4^{\circ} & 60.2^{\circ} \\ 55 \pm 3 \% & 50.3 \% \\ 110 \pm 6^{\circ} & 102.7^{\circ} \\ 104 \pm 2^{\circ} & 96.6^{\circ} \\ 22 \pm 5^{\circ} & 14.8^{\circ} \\ 4 \pm 3 \mathrm{~mm} & 2.1 \mathrm{~mm} \\ 131 \pm 5^{\circ} & 143.1^{\circ} \\ 93 \pm 6^{\circ} & 90.4^{\circ} \\ 25 \pm 6^{\circ} & 22.6^{\circ} \\ 4 \pm 2 \mathrm{~mm} & 1.3 \mathrm{~mm} \\ 1 \pm 2 \mathrm{~mm} & -2.3 \mathrm{~mm} \\ -4 \pm 2 \mathrm{~mm} & -5.1 \mathrm{~mm} \\ -2 \pm 2 \mathrm{~mm} & -4.7 \mathrm{~mm} \\ 100 \pm 10^{\circ} & 88.1^{\circ}\end{array}$




\section{Treatment objectives}

Based on the clinical examination and records analysis, the treatment objectives were:

1. Distalizing the maxillary molars to achieve class I molar and canine relationship bilaterally, normalized overjet and overbite.

2. Align all lower and upper permanent teeth and correct rotated teeth.

3. Correct upper and lower midline and improve soft tissue relationship.

\section{Treatment options}

Based on the clinical examination and treatment objectives, the following treatment plans mentioned:

1. Comprehensive, non-surgical, non-extraction fixed orthodontic treatment by extraction of the upper and lower third molars and distalization of the upper first and second molars by using a Frog Appliance, supported by temporary anchorage device (TADs).

2. Comprehensive, non- surgical, extraction fixed orthodontic treatment by extraction of maxillary first premolars and mandibular second premolars with upper moderate and lower minimum anchorage.

3. After we discussed these options with the patient and her parents, they rejected the second option, agreed on the first option and inform consent signed.

\section{Proposed retention strategy and rationale}

Based on the treatment plan that have been chosen by the patient and her parents, the retention protocol was upper modified Hawley retainer with anterior bite plate and lower fixed retainer extend from first premolar to other first premolar in addition to Hawley retainer. Since this case has deep bite, ideal axial inclination of the incisors at the end of treatment could be a factor in deep bite stability. Proper interincisal angle should be achieved to prevent the deep bite relapse. ${ }^{10}$ Lower fixed retainer will be placed to keep the anterior teeth alignment and to avoid deep bite relapse. Using anterior bite plate with Hawley retainer will prevent relapse of deep bite. Keeping lower incisors in contact with the palatal acrylic of the upper retainer will prevent lower incisors eruption and allow eruption of posterior teeth. ${ }^{11}$ Long-term use of Hawley retainer firmly holds each tooth in its position and maintain transverse width.

\section{Treatment progress}

Treatment started with placement of separators in upper and lower first and second molars and referred the patient to oral surgeon to extract upper and lower third molars. Bonding upper arch 5-5 teeth with preadjusted fixed appliances $(0.022 \times 0.028$-inch, MBT system $)$, band upper first and second molars and leveling and alignment upper arch till reach to $0.019 \mathrm{x} 0.025 \mathrm{Niti}$. Single TAD (OrthoEasy ${ }^{\circledR}$ Pin 0.022 “ $/ 1,7 \times 8 \mathrm{~mm}$ ) Forestadent have been placed in palate area. Alginate impression taken for upper arch to construct modified frog appliance. A frog appliance kit consists of: screw, preformed spring and screw driver (Figure 5).

The manufacturer advice, occluso-apically the frog screw should be placed approximately $10 \mathrm{~mm}$ to $12 \mathrm{~mm}$ from the occlusal surface. This produce bodily movement of the molars because the appliance approximately at the center of resistance of the molars. Lingual sheaths soldered to the lingual surface of upper first molar bands and anterior part of frog appliance solder to miniscrew cap. At the time of appliance insertion, the upper first and second molar bands cemented using a multi-cure glass ionomer orthodontic band cement, inserted the ends of the distalizing spring in to the lingual sheaths of the molar bands, adapt anterior part of appliance in TAD then inserted the spring in posterior part of appliance (Figure 6).

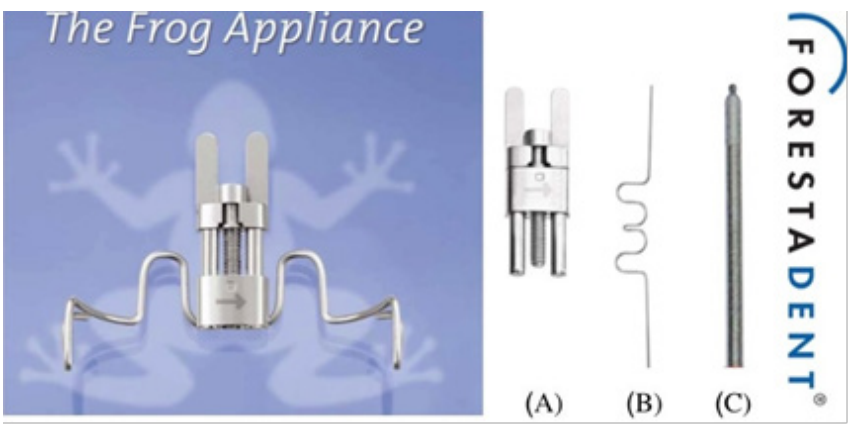

Figure 5 (A) Frog appliance kit (A) screw (B) preformed Spring (C) screw driver.

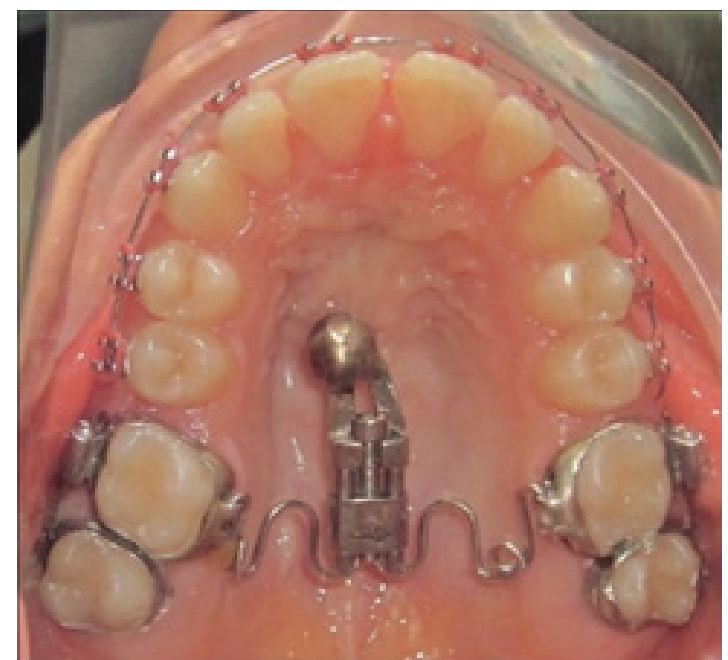

Figure $6 \mathrm{~A}$ frog appliance in its place.

After checking stability of appliance, cut the main arch wire $(0.019 x 0.025 \mathrm{SS})$ between second premolar and first molar bilaterally then start the activation of appliance. According to the manufacturer instruction, one complete rotation around the axis of the activation screw opens the appliance $0.4 \mathrm{~mm}$. Three rotations are recommended for four to five-week interval visit and five rotations are recommended for eight-week interval visit. At each appointment, oral hygiene, the stability of the appliance and progress of distalization were evaluated till super class I molar relationship has achieved. After the maxillary first and second molars distalized, expansion done in the upper arch by open the loops in frog appliance spring wire. Later the device was left in place as a retention appliance for three months. Sectional arch wire has been replaced with continues arch wire $0.016 \times 0.022$ Niti. One month later, start leveling and alignment of lower arch from right second molar to left second molar till reach $(0.019 \times 0.025 \mathrm{SS})$ in both arches and start space closure. At the end of active treatment, finishing procedures were applied for final alignment of the teeth, detailing of the occlusion, frog appliance and TAD had been removed. After 21 months of treatment, debonding of upper and lower arches, retainers delivered for the patient. 


\section{Results}

At the end of orthodontic treatment, bilateral Class I molar and canine relationship was achieved with normal OJ. All upper and lower teeth aligned with flat curve of speed along with coinciding upper and lower teeth midline with the facial midline. Acceptable overbite was attained with alleviation of deep traumatic bite (Figure 7).

Final cephalometric analysis and superimposition of pretreatment and posttreatment tracings showed that the skeletal Class I relationship had been achieved with increase of the lower anterior facial height (Figure $8 \&$ Table 2). Overall superimposition showed nose grew downward by $2 \mathrm{~mm}$, an upper lip moved downward by $2 \mathrm{~mm}$ and backward $3 \mathrm{~mm}$, lower lip moved upward by $2 \mathrm{~mm}$ and backward 1 $\mathrm{mm}$, point A moved backward by $1 \mathrm{~mm}$, point B moved forward 2 $\mathrm{mm}$ and downward $2 \mathrm{~mm}$ based on Bjork method of stable structure that register on the inner contour of the anterior wall of sella turcic. Maxillary and mandibular regional superimposition showed that upper central incisor proclined by $6^{\circ}$ and retruded by $1.5 \mathrm{~mm}$ and intruded by $1 \mathrm{~mm}$ and upper first molar moved distally by $4.5 \mathrm{~mm}$. However lower incisor proclined by $9^{\circ}$ and intruded by $2 \mathrm{~mm}$ and lower first molar mesialized by $2 \mathrm{~mm}$ and extruded $2.5 \mathrm{~mm}$. Downward backward rotation of mandible based on Bjork method of stable structure that register on anterior wall of key ridge and the inner cortical structure at the inferior border of the symphysis and mandible canal (Figure 9). The final panoramic radiograph showed a good root parallelism except \#15, 25 and \#34 dilaceration (Figure 10).
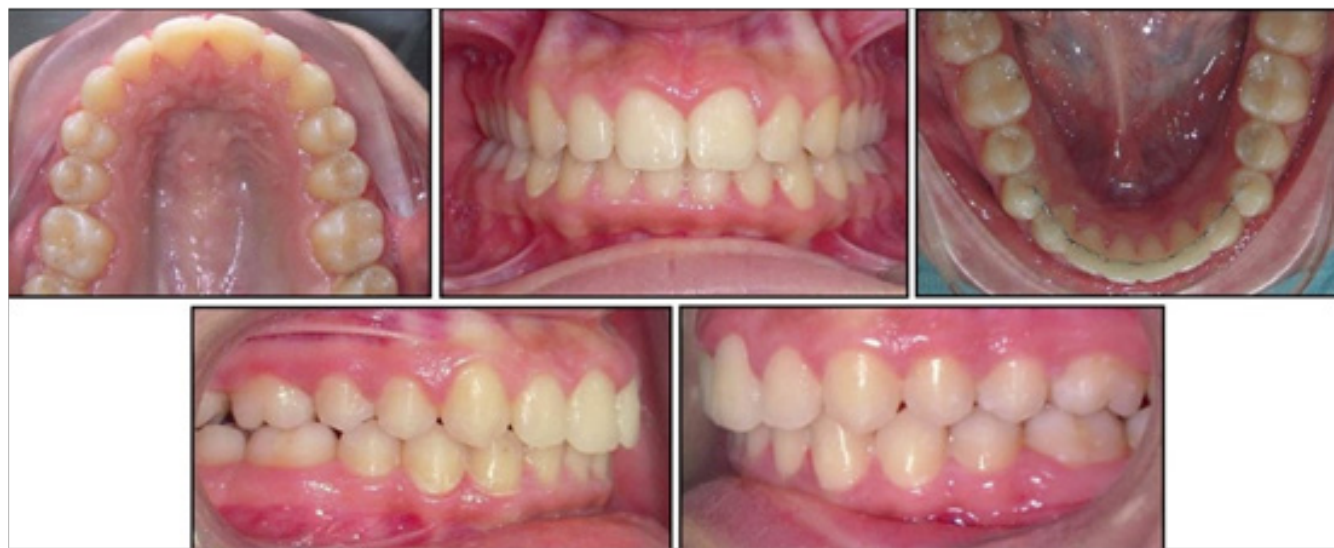

Figure 7 Post-treatment intraoral photographs.

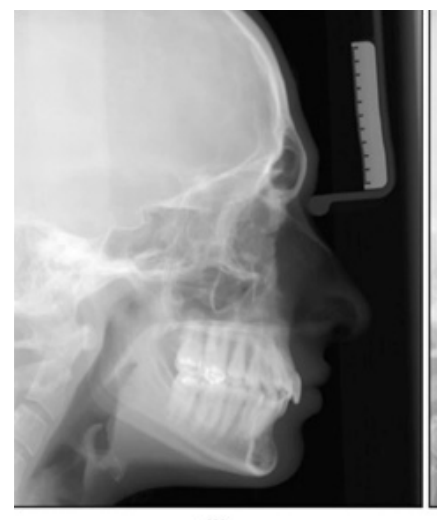

(A)

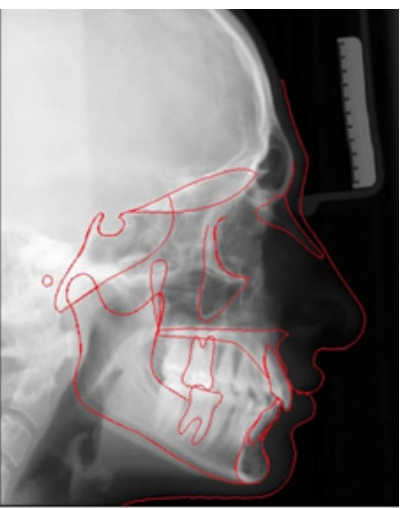

Figure 8 (A) Post-treatment lateral cephalometric radiograph; (B) Posttreatment traced lateral cephalometric radiograph.

Table 2 Post-treatment lateral cephalometric reading

\begin{tabular}{llll}
\hline Measurement & Mean & Pre-treat & Post-treatment \\
\hline SNA & $82 \pm 2^{\circ}$ & $80.9^{\circ}$ & $80.5^{\circ}$ \\
SNB & $80 \pm 2^{\circ}$ & $76.1^{\circ}$ & $77.8^{\circ}$ \\
ANB & $2 \pm 2^{\circ}$ & $4.8^{\circ}$ & $2.7^{\circ}$ \\
NA - APg. & $0 \pm 5^{\circ}$ & $6.1^{\circ}$ & $3.8^{\circ}$ \\
Wits Appraisal & $1.2 \pm 1.9 \mathrm{~mm}$ & $3.5 \mathrm{~mm}$ & $1.3 \mathrm{~mm}$
\end{tabular}

\begin{tabular}{llll} 
Mand. plane to FH & $25 \pm 5^{\circ}$ & $19.3^{\circ}$ & $22.9^{\circ}$ \\
Mand. Plane to SN & $32 \pm 5.1^{\circ}$ & $26.4^{\circ}$ & $30.0^{\circ}$ \\
Max. Plane to SN & $8 \pm 3^{\circ}$ & $10.3^{\circ}$ & $10.9^{\circ}$ \\
Max. to Mand. Plane & $25 \pm 3^{\circ}$ & $21.6^{\circ}$ & $25.1^{\circ}$ \\
Y axis SGn. / SN & $59 \pm 4^{\circ}$ & $60.2^{\circ}$ & $62.9^{\circ}$ \\
Lower Face Height & $55 \pm 3 \%$ & $50.3 \%$ & $53.1 \%$ \\
U Inc. to Max. plane & $110 \pm 6^{\circ}$ & $102.7^{\circ}$ & $108.2^{\circ}$ \\
U Inc. to SN. & $104 \pm 2^{\circ}$ & $96.6^{\circ}$ & $102.1^{\circ}$ \\
U Inc. to NA & $22 \pm 5^{\circ}$ & $14.8^{\circ}$ & $20.3^{\circ}$ \\
U Inc. to NA (mm) & $4 \pm 3 \mathrm{~mm}$ & $2.1^{\circ}$ & $2.9 \mathrm{~mm}$ \\
U Inc. to L Inc. & $131 \pm 5^{\circ}$ & $143.1^{\circ}$ & $131.9^{\circ}$ \\
L Inc. to Mand. & $93 \pm 6^{\circ}$ & $90.4^{\circ}$ & $99.9^{\circ}$ \\
\hline L Inc. to NB & $25 \pm 6^{\circ}$ & $22.6^{\circ}$ & $32.1^{\circ}$ \\
\hline L Inc. to NB (mm) & $4 \pm 2 \mathrm{~mm}$ & $1.3 \mathrm{~mm}$ & $4.3 \mathrm{~mm}$ \\
L Inc. to APog. (mm) & $1 \pm 2 \mathrm{~mm}$ & $-2.3 \mathrm{~mm}$ & $3.5 \mathrm{~mm}$ \\
Upper lip to E-line & $-4 \pm 2 \mathrm{~mm}$ & $-5.1 \mathrm{~mm}$ & $-3.5 \mathrm{~mm}$ \\
Lower lip to E-line & $-2 \pm 2 \mathrm{~mm}$ & $-4.7 \mathrm{~mm}$ & $-1.8 \mathrm{~mm}$ \\
Nasio-labial angle & $100 \pm 10^{\circ}$ & $88.1^{\circ}$ & $90.6^{\circ}$
\end{tabular}




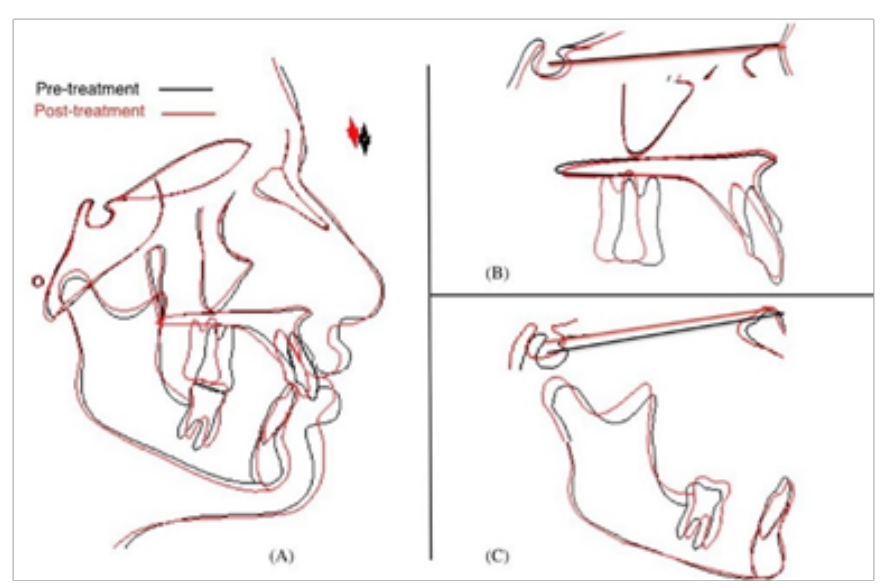

Figure 9 (A) overall superimposition; (B) regional maxillary superimposition; (C) regional mandibular superimposition.

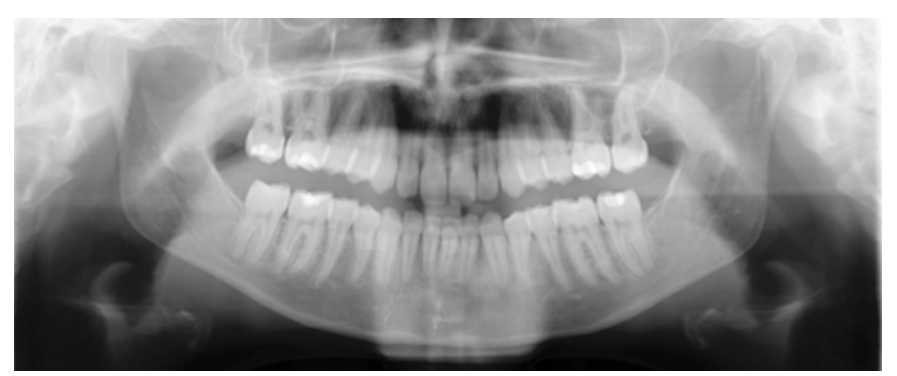

Figure 10 Post-treatment panoramic radiographs.

\section{Discussion}

The patient presented with skeletal Class II due to retrognathic mandible and Class II div 2 malocclusion with decrease lower anterior facial height, reduce mandibular plane angle and traumatic deep bite (short face features). Non extraction treatment favored as this approach takes an advantage of increasing vertical facial height to enhance overbite correction. Extraction in low angle cases complicate the mechanics of overbite correction because strong muscles force impedes the ability of the posterior teeth to move forward. ${ }^{12,13}$ Orthodontists for the most part concur that space closing mechanics tend to deepen the bite and making it more difficult to correct and/or maintain the proper overbite relationship in patients with deep bite. ${ }^{14}$

Molar distalization is recommended for the correction of Class II malocclusions in deep bite patients and in the presence of a convex or normal facial profile. ${ }^{15}$ So, non-extraction with maxillary molars distalization option was offered to the patient as the best option and the patient agreed with it.

The advantages of Frog appliance : Palatal appliance, Skeletal anchorage, Hygienic, 3D movement, the ability to measure the amount of distalization, easy in activation and adjustable arms (not solder to bands).

To eliminate the need for dental anchorage and its undesirable side effects on the anterior arch, we have added TAD within Frog molar distalization appliance to eliminate the need of dental support or acrylic palatal button. The anterior area of the palate is considered to be a safe region for miniscrew insertion because it is far from the teeth roots and other important anatomic structure. ${ }^{16}$

\section{Conclusion}

Miniscrew supported Frog appliance is a fixed appliance, reduce the need of patient compliance. Moreover, the use of this appliance results in successful, distalization of maxillary molars. The outcomes from this case report reflected that the Frog appliance is an effective and a reliable method for the distalization of maxillary molars. The use of miniscrews with this appliance eliminate the effect of distalizer on the other teeth.

\section{Funding}

None.

\section{Acknowledgments}

The authors would like to thank Dr.Abdulaziz K. AlKhunain, consultant in orthodontics, teaching faculty member at prince Abdurrahman advance dental institute Riyadh, Saudi Arabia for all of his careful, constructive and insightful comments in relation to this work.

\section{Conflicts of interest}

The authors declare there are no conflicts of interest.

\section{References}

1. Al-Emran S, Wisth P, Boe O. Prevalence of malocclusion and need for orthodontic treatment in Saudi Arabia. Community Dentistry and Oral Epidemiology. 1990;18(5):253-255.

2. Brezniak N, Arad A, Heller M, et al. Pathognomonic cephalometric characteristics of Angle Class II Division 2 malocclusion. Angle Orthod. 2002;72(3):251-57.

3. Atik E, Kocadereli I. Treatment of Class II Division 2 Malocclusion Using the Forsus Fatigue Resistance Device and 5-Year Follow-Up. Case Reports in Dentistry. 2016;2016:1-7.

4. Dodda K, Prasad S, Kanuru R, et al. Diagnostic features of Angle's Class II div 2 malocclusion. Journal of International Society of Preventive and Community Dentistry. 2015;5(6):513.

5. Bock N, Ruf S. Post-treatment occlusal changes in Class II division 2 subjects treated with the Herbst appliance. The European Journal of Orthodontics. 2008;30(6):606-613.

6. Hilgers JJ. The pendulum appliance for Class II non-compliance therapy. J Clin Orthod. 1992;26(11):706-714.

7. Fortini A, Lupoli M, Giuntoli F, et al. Dentoskeletal effects induced by rapid molar distalization with the first class appliance. American Journal of Orthodontics and Dentofacial Orthopedics. 2004;125(6):697-704.

8. Bayram M, Nur M, Kilkis D. The frog appliance for upper molar distalization: a case report. The Korean Journal of Orthodontics. 2010;40(1):50

9. Park HS, Lee SK, Kwon OW. Group distal movement of teeth using microscrew implant anchorage. Angle Orthod. 2005; 75:602-609.

10. Zachrisson BU. Important aspects of long-term stability. J Clin Orthod. 1997;31:562-83.

11. Proffit W, Fields H, Larson B, Sarver D. Contemporary orthodontics. 5th ed. Mosby: St Louis; 2012.

12. Abdo Quintao C, Miguel J, Brunharo I, et al. Is traditional treatment a good option for an adult with a Class II deepbite malocclusion? American Journal of Orthodontics and Dentofacial Orthopedics. 2012;141(1):105-112. 
13. Moss JP. An investigation of the muscle activity of patients with Class II Division 2 malocclusion and the changes during treatment. Trans Eur Orthod Soc. 1975:87-101.

14. Cole HJ. Certain results of extraction in the treatment of malocclusion Angle Orthod. 1948;18:103-13.
15. Sfondrini M, Cacciafesta V, Sfondrini G. Upper molar distalization: a critical analysis. Orthodontics and Craniofacial Research. 2002;5(2):114-126.

16. Gelgör IE, Büyükyilmaz T, Karaman AI, et al. Intraosseous screwsupported upper molar distalization, Angle Orthod. 2004;74(6):838850. 